\title{
Clinical Decision Support Systems and Computerized Provider Order Entry: Contributions from 2020
}

\author{
Damian Borbolla', Grégoire Ficheur', Section Editors for the IMIA Yearbook Section on \\ Decision Support \\ 1 Department of Biomedical Informatics, University of Utah, Salt Lake City, UT, USA \\ 2 Univ. Lille, CHU Lille, ULR 2694 - METRICS, Public health dept, Lille, France
}

\begin{abstract}
Summary
Objectives: To summarize research contributions published in 2020 in the field of clinical decision support systems (CDSS) and computerized provider order entry (CPOE), and select the best papers for the Decision Support section of the International Medical Informatics Association (IMIA) Yearbook 2021.

Methods: Two bibliographic databases were searched for papers referring to clinical decision support systems. From search results, section editors established a list of candidate best papers, which were then peer-reviewed by seven external reviewers. The IMIA Yearbook editorial committee finally selected the best papers on the basis of all reviews including the section editors' evaluation. Results: A total of 1,919 articles were retrieved. 15 best paper candidates were selected, the reviews of which resulted in the selection of two best papers. One paper reports on the use of electronic health records to support a public health response to the COVID-19 pandemic in the United States. The second paper proposes a combination of CDSS and telemedicine as a technology-based intervention to improve the outcomes of depression as part of a cluster trial.

Conclusions: As shown by the number and the variety of works related to clinical decision support, research in the field is very active. This year's selection highlighted the application of CDSS to fight COVID-19 and a combined technology-based strategy to improve the treatment of depression.
\end{abstract}

\section{Keywords}

Health informatics, International Medical Informatics Association, Review, clinical decision support systems

Yearb Med Inform 2021:172-5

http://dx.doi.org/10.1055/s-0041-1726534

\section{Introduction}

This paper serves as the synopsis of the decision support section of the International Medical Informatics Association (IMIA) Yearbook 2021. It complements the survey paper authored by Taber et al. where the authors sought to identify the current state of research of clinical decision support system (CDSS) using interoperability standards [1]. Our aim is to summarize recent research in the domain of decision support and to select the best papers published in this field in 2020. This literature review focused on research studies related to CDSS and computerized provider order entry (CPOE) systems. The synopsis is organized as follows: the next section summarizes the process for selecting the best papers on the decision support topic; then we present the results of this year's selection process, and the last section comments the contributions of the two best papers, as well as noticeable research works in the field identified during the whole process.

\section{Methods}

A comprehensive literature search on topics related to CDSSs and CPOE systems was performed to identify candidate best papers in two bibliographic databases, the PubMed/Medline database (from the US National Center for Biotechnology Information) and the Web of Science ${ }^{\circledR}$ (WoS, from Clarivate Analytics). Both databases were searched with similar queries, tailored to the specificities of each one, targeting journal articles published in 2020, written in English, and related to the topics of interest. The adopted strategy was the one described in the paper of Lamy et al. [2].

A first review of the retrieved citations was performed by the two section editors to select 15 candidate best papers. Following the IMIA Yearbook protocol, these candidate best papers were then individually reviewed and rated by both section editors, the chief editor of the Decision Support section, and external reviewers from the international Health Informatics community. Based on the reviewers' ratings and comments, the Yearbook editorial committee then selected the best papers of the decision support domain.

\section{Results}

A total of 1,919 unique references were obtained. After a first individual screening independently performed by both section editors based on the title of papers, 1,816 were rejected by both of them. 103 studies were then discussed by the two editors to achieve a final selection of 15 candidate best papers. After the external review of these 15 articles, the editorial committee finally selected two of them as best papers for 2020. Table 1 summarizes 15 papers initially selected by the section editors. The two best papers of 2020 are discussed in the next section. 
Table 1 The 15 papers initially selected

- J Jeffery Reeves et al. Rapid response to COVID-19: health informatics support for outbreak management in an academic health system. J Am Med Inform Assoc 2020 Jun 1;27(6):853-859. doi: 10.1093/jamia/ocaa037. PMID: 32208481; PMCID: PMC7184393.

- Elisha S Grange et al. Responding to COVID-19: The UW Medicine Information Technology Services Experience. Appl Clin Inform 2020 Mar; 11(2):265-275. doi: 10.1055/s-0040-1709715. Epub 2020 Apr 8. PMID: 32268390; PMCID: PMC7141898.

- Guangyao Wu et al. Development of a clinical decision support system for severity risk prediction and triage of COVID-19 patients at hospital admission: an international multicentre study. Eur Respir J 2020 Aug 20;56(2):2001 104. doi: 10.1183/13993003.01104-2020. PMID: 32616597; PMCID: PMC7331655

- Mengchun Gong et al. Cloud-Based System for Effective Surveillance and Control of COVID-19: Useful Experiences From Hubei, China. J Med Internet Res 2020 Apr 22;22(4):e18948. doi: 10.2196/18948. PMID: 32287040; PMCID: PMC7179239.

- Paul M McKie et al. Computerized Advisory Decision Support for Cardiovascular Diseases in Primary Care: A Cluster Randomized Trial. Am J Med 2020 Jun;133(6):750-756.e2. doi: 10.1016/i.amjmed.2019.10.039. Epub 2019 Dec 18. PMID: 31862329.

- Jennifer Corny et al. A machine learning-based clinical decision support system to identify prescriptions with a high risk of medication error. J Am Med Inform Assoc 2020 Nov 1;27(11):1688-1694. doi: 10.1093/jamia/ocaa154. PMID: 32984901; PMCID: PMC7671619.

- Subha Madhavan et al. Use of electronic health records to support a public health response to the COVID-19 pandemic in the United States: a perspective from 15 academic medical centers. J Am Med Inform Assoc 2021 Feb 15;28(2):393-401. doi: 10.1093/jamia/ocaa287. PMID: 33260207; PMCID: PMC7665546.

- David Gallagher et al. Implementation and Continuous Monitoring of an Electronic Health Record Embedded Readmissions Clinical Decision Support Tool. J Pers Med 2020 Aug 26;10(3):103. doi: 10.3390/ipm10030103. PMID: 32858890; PMCID: PMC7565687.

- Nicolas Delvaux et al. Clinical decision support improves the appropriateness of laboratory test ordering in primary care without increasing diagnostic error: the ELMO cluster randomized trial. Implement Sci 2020 Nov 4;15(1):100. doi: 10.1186/s13012020-01059-y. PMID: 33148311; PMCID: PMC7640389.

- Peter G Szilagyi et al. Effect of Patient Portal Reminders Sent by a Health Care System on Influenza Vaccination Rates: A Randomized Clinical Trial. JAMA Intern Med 2020 Jul 1;180(7):962-970. doi: 10.1001/jamainternmed.2020.1602. PMID: 32421168; PMCID: PMC7235900.

- Rebecca L Curran et al. Integrated displays to improve chronic disease management in ambulatory care: A SMART on FHIR application informed by mixed-methods user testing. J Am Med Inform Assoc 2020 Aug 1;27(8):1225-1234. doi: 10.1093/ jamia/ocaa099. PMID: 32719880; PMCID: PMC7481023.

- Joseph A Greer et al. Randomized Trial of a Smartphone Mobile App to Improve Symptoms and Adherence to Oral Therapy for Cancer. J Natl Compr Canc Netw 2020 Feb;18(2):133-141. doi: 10.6004/inccn.2019.7354. PMID: 32023526.

- Jasmeet $S$ Dhaliwal et al. Reduced admission rates and resource utilization for chest pain patients using an electronic health record-embedded clinical pathway in the emergency department. J Am Coll Emerg Physicians Open 2020 Nov 17;1(6):16021613. doi: 10.1002/emp2.12308. PMID: 33392569; PMCID: PMC7771814.

- Jessica P Ridgway et al. A Randomized Controlled Trial of an Electronic Clinical Decision Support Tool for Inpatient Antimicrobial Stewardship. Clin Infect Dis 2021 May 4;72(9):e265-e271. doi: 10.1093/cid/ciaa1048. PMID: 32712674.

- Matteo Balestrieri et al. Effectiveness of clinical decision support systems and telemedicine on outcomes of depression: a cluster randomized trial in general practice. Fam Pract 2020 Nov 28;37(6):731-737. doi: 10.1093/fampra/cmaa077. PMID: 32766705.

Table 2 Best paper selection of articles for the IMIA Yearbook of Medical Informatics 2021 in the special section 'Decision Support'. The articles are listed in alphabetical order of the first author's surname.

\section{Section}

Decision Support

- Balestrieri M, Sisti D, Rocchi M, Rucci P, Simon G, Araya R, de Girolamo G. Effectiveness of clinical decision support systems and telemedicine on outcomes of depression: a cluster randomized trial in general practice. Fam Pract 2020 Nov 28;37(6):731-7.

- Wu G, Yang P, Xie Y, Woodruff HC, Rao X, Guiot J, Frix AN, Louis R, Moutschen M, Li J, Li J, Yan C, Du D, Zhao S, Ding Y, Liu B, Sun W, Albarello F, D’Abramo A, Schininà V, Nicastri E, Occhipinti M, Barisione G, Barisione E, Halilaj I, Lovinfosse $P$, Wang $X$, Wu J, Lambin P. Development of a clinical decision support system for severity risk prediction and triage of COVID-19 patients at hospital admission: an international multicentre study. Eur Respir J 2020 Aug 20;56(2):2001104.

\section{Discussion and Outlook}

The first paper, by Wu et al. [3], proposed a clinical decision support system for severity risk prediction of COVID-19 patients at hospital admission. The model was built in a supervised manner from 725 patients in order to predict the onset of severe or critical illness during hospitalization. It was an international multicenter study including retrospective and prospective cohorts from China, Italy and Belgium. The final list of features included in the model were age, lymphocytes (proportion), CRP, LDH, creatine kinase, urea and calcium. This modelisation is proposed as an online calculator tool, as well as transcribed through a nomogram and trial tool for clinicians. The very early conduct of this large-scale multicenter study during the epidemic, as well as the pragmatism of the dissemination of the model through these tools, seems to the editors to be highlighted.

In the second paper, Balestrieri et al. [4] aimed to test the effectiveness of a composite CDSS and telemedicine combined approach designed to treat depression in primary care. This intervention was assessed through a cluster randomized trial in four clinics in Italy comparing the technology-based intervention versus usual treatment. The algorithm used in this project was a computerized treatment system developed by expert consensus; it provided all prompts necessary to guide the GPs in the choice of the best treatment and provided guidance about the need of a referral to a specialist service. The percentage of remitters at 6 months was significantly lower in the intervention group ( $24.1 \%$ vs $6.6 \%)$. This work seems to us to be a reference article for the global evaluation of a decision support tool including a content and an expert decision tree. The study design, always a challenge when implementing CDSS interventions, used in this article is an excellent example of how CDSS can be disseminated.

Besides the two best papers selected for the Decision Support section of the 2021 edition of the IMIA Yearbook, several other works deserve to be cited. One paper describes the use of electronic health records to support a public health response to the COVID-19 pandemic with a focus 
on academic centers in the United States [5]. Other papers focus on the integration of Artificial Intelligence (AI) and machine learning-based with clinical decision support system to help in different clinical situations [6].

As showed in the amount and the variety of studies found in this synopsis, research in the field of CDSS is very active. As expected, this year's selection highlighted the impact that COVID-19 and the global crisis had on public health and how information system and especially CDSS can help to implement technology-based solutions. Telehealth and machine learning tools associated to CDSS were also emerging in the scientific literature.

\section{References}

1. Taber P, Radloff C, Del Fiol G, Staes C, Kawamoto K. New Standards for Clinical Decision Support: A Survey of The State of Implementation. Yearb Med Inform 2021:159-71.

2. Lamy JB, Séroussi B, Griffon N, Kerdelhué G, Jaulent MC, Bouaud J. Toward a formalization of the process to selec IMIA Yearbook best papers. Methods Inf Med. 2015;54(2):135-44.

3. Wu G, Yang P, Xie Y, Woodruff HC, Rao X, Guiot $J$, et al. Development of a clinical decision support system for severity risk prediction and triage of COVID-19 patients at hospital admission: an international multicentre study. Eur Respir J 2020 Aug 20;56(2):2001104.

4. Balestrieri M, Sisti D, Rocchi M, Rucci P, Simon G, Araya R, et al. Effectiveness of clinical decision support systems and telemedicine on outcomes of depression: a cluster randomized trial in general practice. Fam Pract 2020 Nov 28;37(6):731-7.

5. Madhavan S, Bastarache L, Brown JS, Butte AJ, Dorr DA, Embi PJ, et al. Use of electronic health records to support a public health response to the COVID-19 pandemic in the United States: a perspective from 15 academic medical centers. JAm Med Inform Assoc 2021 Feb 15;28(2):393-401.

6. Corny J, Rajkumar A, Martin O, Dode X, Lajonchère JP, Billuart $\mathrm{O}$, et al. A machine learning-based clinical decision support system to identify prescriptions with a high risk of medication error. J Am Med Inform Assoc 2020 Nov 1;27(11):1688-94.

\author{
Correspondence to: \\ Damian Borbolla \\ University of Utah, Salt Lake City, UT, USA \\ E-mail: Damian.borbolla@utah.edu
}




\section{Appendix: Content Summa- ries of Best Papers for the Decision Support Section of the 2021 IMIA Yearbook}

\section{Wu G, Yangx AN, Louis R, Moutschen $M$, Li J, Li J, Yan C, Du D, Zhao S, Ding Y, Liu B, Sun W, Albarello F, D'Abramo A, Schininà V, Nicastri E, Occhipinti M, Barisione G, Barisione $E$, Halilaj I, Lovinfosse P, Wang $X$, Wu J, Lambin P \\ Development of a clinical decision support system for severity risk prediction and triage of COVID-19 patients at hospital admission: an international multicentre study}

\section{Eur Respir J 2020 Aug 20;56(2):2001104}

In this paper the authors aimed to develop a machine learning algorithm to assess and triage patients with COVID-19. They used 725 patients to train the model, including cohorts from hospitals in China, Italy and Belgium. The main outcome was the onset of severe or critical illness during hospitalization. Model performances were quantified using the area under the receiver operating characteristic curve (AUC) and other metrics. The model was validated yielding AUCs ranging from 0.84 to 0.93 , with accuracies ranging from $74.4 \%$ to $87.5 \%$, sensitivities ranging from $75.0 \%$ to $96.9 \%$, and specificities ranging from $55.0 \%$ to $88.0 \%$, most of which performed better than the pneumonia severity index. The authors concluded that the machine-learning algorithm is useful to triage the severity and critical illness among COVID-19 patients at hospital admission. The online calculators can be found at www.covid19risk.ai.

\section{Balestrieri M, Sisti D, Rocchi M, Rucci P, Simon G, Araya R, de Girolamo G \\ Effectiveness of clinical decision support systems and telemedicine on outcomes of}

depression: a cluster randomized trial in general practice

\section{Fam Pract 2020 Nov 28;37(6):731-7}

In this paper, the authors combined a Computerized Clinical Decision Support Systems (CCDSS) tool with a Telehealth Intervention and tested the effectiveness of this combined approach to trat depression in primary care. The design was a randomized trial involving General Practice clinics in Italy. The study compared the combined Intervention (TG - Telehealth visit + CCDSS) with the control group (CG) in which GPs provided usual treatment. 2810 patients were included. 66 patients were included in the TG group and 32 in the CG. The percentage of remitters at 6 months was significantly higher in the TG than in the CG group (24.1\% versus $3.1 \%, \chi 2=6.6, \mathrm{P}=0.01)$. The combined intervention was more effective than the usual care offered by GPs to patients with depression in the study. 